\title{
Visual Field Protective Effect of Erigeron breviscapus (vant.) Hand. Mazz. Extract on Glaucoma with Controlled Intraocular Pressure
}

\section{A Randomized, Double-Blind, Clinical Trial}

Yisheng Zhong, ${ }^{1}$ Minhong Xiang, ${ }^{1}$ Wen Ye, ${ }^{2}$ Yu Cheng ${ }^{1}$ and Youqin Jiang ${ }^{3}$

1 Department of Ophthalmology, Ruijin Hospital Affiliated Medical School, Shanghai Jiaotong University, Shanghai, China

2 Department of Ophthalmology, Huashan Hospital Affiliated Fudan University, Shanghai, China

3 Department of Ophthalmology, Xiangya Second Hospital Affiliated Central South University, Changsha, China

Objective: To evaluate the visual field protective effect of Erigeron breviscapus (vant.) Hand. Mazz. (EBHM) extract on glaucoma with controlled intraocular pressure (IOP).

Methods: Forty patients (40 eyes) with primary open-angle glaucoma, visual field defects and a postsurgical IOP of $<18 \mathrm{mmHg}$ were enrolled. The EBHM and placebo tablets were given orally according to the randomized and double-blind principle. Two tablets (of either EBHM or placebo) were taken three times a day for a period of 6 months. Patients were examined every 2 months after treatment commenced. At the end of the study, the results were given to the drug manufacturer.

Results: All patients completed the prospective, randomized, double-blind, clinical trial. No obvious adverse effects were found in patients during the treatment period. In the placebo group, no significant difference was found in mean defect (MD) or mean sensitivity (MS) between the values at pretreatment and after 2, 4, and 6 months of treatment. After 6 months of EBHM treatment, the MD was significantly decreased and the MS was significantly increased compared with pre-treatment $(\mathrm{p}<0.05)$. In the patients with moderate and late glaucoma, the MD was significantly decreased and the MS was significantly increased after 2, 4, and 6 months of EBHM treatment compared with pre-treatment.

Conclusion: EBHM extract may have a partial protective effect on the visual field of glaucoma patients with controlled IOP. Further studies are needed to determine the safety and effectiveness of long-term EBHM treatment. 


\section{Introduction}

Glaucoma is a progressive optic neuropathy involving characteristic structural pathologic changes in the optic nerve head. ${ }^{[1-3]}$ Reducing intraocular pressure (IOP) is presently the evidence-based, most accepted and most practiced therapeutic approach for glaucoma patients. ${ }^{[4-6]}$ However, progressive loss of visual field or blindness can still occur in some glaucoma patients with controlled IOP. ${ }^{[7]}$ It is now recognized that the major detrimental effect of glaucoma is destruction of retinal ganglion cells (RGCs). Therefore, in the management of glaucoma, neuroprotection-based therapies are the most important.

Erigeron breviscapus (vant.) Hand. Mazz. (EBHM) [Deng-zhan-hua in Chinese] is a well known traditional Chinese medicinal plant for heart disease. ${ }^{[8]}$ Previous pharmacologic studies demonstrated that EBHM could stimulate the fibrinolytic and anticoagulant properties of endothelial cells, dilate blood vessels, reduce blood vessel resistance, increase blood flow, improve microcirculation, and inhibit thrombosis. ${ }^{[9,10]}$ Previous studies have shown that EBHM could improve the activity of cytochrome oxidase in RGCs and optic nerve axoplasmic transport in rat models of acute elevated IOP. ${ }^{[1,12]}$ In 2001, the China State Drug Administration approved EBHM extract for clinical use. In order to evaluate the effectiveness of EBHM extract in glaucoma patients, we conducted a prospective, randomized, double-blind clinical trial in a group of glaucoma patients with controlled IOP.

\section{Patients and Methods}

The study was approved by the institutional review board of Ruijin Hospital (Shanghai, China) and was conducted in accordance with the Declaration of Helsinki. Informed consent was obtained from all patients included in the study. Forty primary open-angle glaucoma (POAG) patients ( 25 men and 15 women) were recruited from the glaucoma specialist clinic of Ruijin Hospital between March 2002 and January 2003. One eye from each patient was chosen for the study. If both of the patient's eyes met the elig- ibility criteria, then one eye was chosen at random. However, patients were also included in the study if only one of their eyes met the eligibility criteria.

Patients were included in this study if they (i) met the POAG diagnosis criteria, namely, a typical glaucomatous abnormal optic nerve head associated with a typical glaucomatous visual field loss (standard automated perimetry), openangle at gonioscopy, a presurgical IOP of $>21 \mathrm{mmHg}$ with no treatment, and no clinically apparent secondary cause for their glaucoma; ${ }^{[13]}$ (ii) had undergone anti-glaucoma surgeries at least 3 months ago, and the IOP was $\leq 18 \mathrm{mmHg}$ with or without medications at two measurements (10am and $3 \mathrm{pm}$ ); (iii) had a refractive error that ranged from -6 to +3 diopters and a clear ocular media with the best-corrected visual acuity of 0.8 or better; (iv) had no clinically apparent retinal disease that might influence the visual field loss, including diabetic retinopathy, macular degeneration, and retinal vascular occlusion; (v) had stopped taking medications that may help the recovery of visual functions for at least 2 months prior, including adenosine triphosphate, inosine, red sage root, and vitamins; (vi) had no mental and nervous system diseases; and (vii) were willing to take part in this trial, and attend follow-up sessions according to the trial design.

Patients were excluded from the trial according to the following criteria: (i) history of serious systemic diseases, such as primary hypertension, heart diseases, gastric ulcer, or diabetes mellitus; (ii) inability or unwillingness to provide informed consent or abide by the study design; (iii) IOP could not be reduced to $18 \mathrm{mmHg}$ or less by medications; (iv) presence of hypotony (IOP $\leq 6 \mathrm{mmHg}$ ) with thin filtering cystic bleb or bleb leakage; (v) end-stage glaucoma that could be evaluated by standard automated perimetry (reliable factor $>15 \%$ ); or (vi) adverse effects due to treatment medication as assessed by a physician.

All patients' baseline data were recorded, including visual acuity, IOP, vertical cup-disk ratio (VCDR), mean defect (MD), mean sensitivity (MS), loss variance (LV), pulse, systolic blood pressure, and diastolic blood pressure. The visual field was assessed by Octopus 101 automated 
perimetry (Interzeg INC, Switzerland), dG2 program full threshold with Dynamic/Normal strategy. The test parameters were as follows: the background luminance was 4 apostilbs, Goldmann size III stimulus was used for the stimulus target, and the target duration time was 100 milliseconds. The crosshair in the screen provided a central fixation mark. All patients had a background illumination adaptation for 5-10 minutes before visual field examination. The visual field examinations were performed under the natural pupil size. If there was presbyopia present, it was corrected using proper convex lenses. Data analysis was derived from all 59 single data points in the central 30 degrees of the visual field. One of the following criteria was used to establish abnormal visual field results: ${ }^{[13]}$ (i) three adjacent points depressed by $5 \mathrm{~dB}$, with one of the points depressed by at least $10 \mathrm{~dB}$; (ii) two adjacent points depressed by $10 \mathrm{~dB}$; or (iii) a $10 \mathrm{~dB}$ difference across the nasal horizontal meridian in two adjacent points. None of the points could be edge points unless immediately above or below the nasal horizontal meridian. ${ }^{[14,15]}$ In addition, visual field testing was only considered reliable when the reliable factor was less than $15 \%$.

POAG was classified as early, moderate and late based on patients' visual field defects. ${ }^{[16]}$ Early POAG patients had an early glaucomatous visual field loss defined as an MD of $\leq 6 \mathrm{~dB}$; moderate POAG patients had a moderate glaucomatous visual field loss defined as $12 \mathrm{~dB} \geq \mathrm{MD}>6 \mathrm{~dB}$; and late POAG patients had a late glaucomatous visual field loss defined as an MD of $>12 \mathrm{~dB}$.

Both EBHM and placebo tablets were similar looking and were provided by Xiangya Pharmaceutical Co. (Mei Er Rui, Changsha, China). The patients received two oral tablets of EBHM (containing $40 \mathrm{mg}$ flavone in each tablet) or placebo, three times a day for a period of 6 months. The EBHM dose used in this study was based on that used in the treatment of cardiovascular disease. Patients were examined every 2 months after treatment commenced. The examination included the following: (i) tests for visual acuity, IOP, VCDR, visual field, pulse, systolic blood pressure, diastolic blood pressure; (ii) a complete physical examination; and (iii) screening for gastric and other adverse effects of the drug.

Statistical analyses were performed using the SAS software package (version 6.12). Student's t-test was used for numerical data and a chi-square test was used for categorical data for comparing the EBHM and placebo groups. To compare data among three or more groups, one-way ANOVA and the Kruskal-Wallis test were used. If a significant difference was detected using ANOVA, Bonferroni correction was used for the post hoc test. The chi-squared test was used for comparison of the visual field improvement rate between the pre- and post-treatment values. A $p$-value of $<0.05$ was considered to be statistically significant.

\section{Results}

A total of 40 patients were recruited in this study, all of whom completed the trial. None of the patients had gastrointestinal discomfort, palpitation, shortness of breath, dizziness or tinnitus. Table I shows the demographic and clinical characteristics of the patients. Baseline visual acuity, IOP, VCDR, pulse, systolic blood pressure, diastolic blood pressure, MD, MS, or LV did not differ significantly between the EBHM and placebo groups.

No significant difference was found in visual acuity, IOP, VCDR, pulse, systolic blood pressure, or diastolic blood pressure between pre- and post-treatments in the EBHM and placebo groups (tables II and III).

No significant difference was found in MD, MS, or LV between pre-treatment and after 2, 4, and 6 months of treatment in the placebo group (table IV). No significant difference was also found in MD, MS, or LV between pre-treatment and 2 and 4 months post-treatment in the EBHM group (table IV). However, after 6 months of EBHM treatment, the MD was significantly decreased and the MS was significantly increased compared with pre-treatment (table IV) [p $<0.05]$.

In the moderate and late glaucoma patients, no significant difference was found in MD, MS, or LV between pre-treatment and after 2, 4, and 6 months of treatment in the placebo group 
Table I. Demographic and clinical characteristics of the patients ${ }^{a}$

\begin{tabular}{lllcc}
\hline Characteristic & EBHM group $(\mathrm{n}=20)$ & Placebo group $(\mathrm{n}=20)$ & $\mathrm{t}$-test or $\chi^{2}$ value & $\mathrm{p}$-Value \\
\hline Age $(\mathrm{y})$ & $63.00 \pm 14.45$ & $64.83 \pm 13.03$ & 0.61 & 0.55 \\
Sex (n [male/female]) & $10 / 10$ & $15 / 5$ & 1.60 & 0.10 \\
Eye (n [OD/OS]) & $10 / 10$ & $11 / 9$ & 1.63 & 0.75 \\
Stage (n [early/moderate or late]) & $8 / 12$ & $5 / 15$ & 0.76 & 0.31 \\
Systolic BP (mmHg) & $127.92 \pm 18.15$ & $133.33 \pm 16.42$ & 0.85 & 0.45 \\
Diastolic BP (mmHg) & $77.92 \pm 9.64$ & $81.25 \pm 9.56$ & 0.63 & 0.40 \\
Pulse (pulses/minute) & $73.08 \pm 12.13$ & $76.42 \pm 13.59$ & 0.53 & 0.53 \\
Visual acuity & $0.79 \pm 0.27$ & $0.79 \pm 0.29$ & -0.79 & 0.47 \\
IOP (mmHg) & $14.33 \pm 2.39$ & $13.71 \pm 2.61$ & -0.44 & 0.43 \\
VCDR & $0.87 \pm 0.17$ & $0.85 \pm 0.15$ & 0.21 & 0.67 \\
MD (dB) & $11.44 \pm 8.84$ & $11.97 \pm 7.32$ & -0.23 & 0.84 \\
MS (dB) & $15.48 \pm 8.46$ & $14.91 \pm 7.60$ & 0.49 & 0.82 \\
LV (dB $)$ & $42.88 \pm 35.82$ & $47.70 \pm 25.06$ & & \\
\hline
\end{tabular}

a Data are expressed as mean $\pm S D$, unless otherwise stated.

$\mathbf{B P}=$ blood pressure; $\mathbf{E B H M}=$ Erigeron breviscapus (vant.) Hand. Mazz; $\mathbf{I O P}=$ intraocular pressure; $\mathbf{L V}=$ loss variance; $\mathbf{M D}=$ mean defect; MS = mean sensitivity; OD=oculus dexter (right eye); OS=oculus sinister (left eye); VCDR=vertical cup-disk ratio.

(table V). However, after 2, 4, and 6 months of EBHM treatment, the MD was significantly decreased and the MS was significantly increased compared with pre-treatment (table V).

The differences in MD values were calculated between pre-treatment and after 2, 4, and 6 months of treatment. A decrease of $>2 \mathrm{~dB}$ in MD was defined as visual field improvement; a change in MD of $\pm 2 \mathrm{~dB}$ was defined as no visual field change; and an increase of $>2 \mathrm{~dB}$ in MD was defined as the visual field retrogression. Table VI shows the visual field improvement rate in the placebo and EBHM groups. After 6 months of treatment, the visual field improvement rate in the EBHM group (35\%) was higher than that in the placebo group (15\%), but did not reach statistical significance. Table VII shows the visual field improvement rates for moderate and late glaucoma in the placebo and EBHM groups. After 6 months of treatment, the visual field improvement rate in the EBHM group (50\%) was higher than that in the placebo group (13.33\%), but again did not reach statistical significance $(\mathrm{p}>0.05)$.

\section{Discussion}

A major goal of glaucoma research has been to develop analogous treatment approaches to prevent the death of ganglion cells of the retina. Risk

Table II. Comparison of blood pressure (BP), pulse, intraocular pressure (IOP), visual acuity, and vertical cup-disk ratio (VCDR) between the pre- and the post-treatment periods in the placebo group $(n=20)^{a}$

\begin{tabular}{|c|c|c|c|c|c|c|}
\hline \multirow[t]{2}{*}{$\overline{\text { Variable }}$} & \multirow[t]{2}{*}{ Pre-treatment } & \multicolumn{3}{|l|}{ Post-treatment } & \multirow[t]{2}{*}{$F$ or $H$ value } & \multirow[t]{2}{*}{$\overline{p-V a l u e}$} \\
\hline & & 2 months & 4 months & 6 months & & \\
\hline Systolic BP (mmHg) & $133.33 \pm 16.42$ & $129.17 \pm 16.49$ & $127.92 \pm 13.73$ & $130.83 \pm 14.28$ & 0.28 & 0.84 \\
\hline Diastolic BP (mmHg) & $81.25 \pm 9.56$ & $80.83 \pm 7.33$ & $82.08 \pm 5.42$ & $81.67 \pm 6.51$ & 0.06 & 0.98 \\
\hline Pulse (pulses/minute) & $76.42 \pm 13.59$ & $79.42 \pm 11.46$ & $77.50 \pm 9.48$ & $76.00 \pm 9.37$ & 0.23 & 0.88 \\
\hline Visual acuity & $0.79 \pm 0.29$ & $0.87 \pm 0.22$ & $0.86 \pm 0.18$ & $0.85 \pm 0.24$ & $9.68^{b}$ & 0.97 \\
\hline IOP (mmHg) & $13.71 \pm 2.61$ & $14.45 \pm 2.19$ & $14.93 \pm 2.14$ & $14.44 \pm 2.69$ & 0.87 & 0.46 \\
\hline VCDR & $0.85 \pm 0.15$ & $0.85 \pm 0.15$ & $0.85 \pm 0.15$ & $0.86 \pm 0.16$ & 0.01 & 1.00 \\
\hline \multicolumn{7}{|c|}{ a $\quad$ Data are expressed as mean \pm SD. } \\
\hline \multicolumn{7}{|c|}{ b Kruskal-Wallis test. } \\
\hline
\end{tabular}


Table III. Comparison of blood pressure (BP), pulse, intraocular pressure (IOP), visual acuity, and vertical cup-disk ratio (VCDR) between the pre- and post-treatment periods in the Erigeron breviscapus (vant.) Hand. Mazz. group $(\mathrm{n}=20)^{\mathrm{a}}$

\begin{tabular}{lllllll}
\hline Variable & Pre-treatment & \multicolumn{2}{l}{ Post-treatment } & \multirow{2}{*}{ For $H$ value } & p-Value \\
\cline { 2 - 5 } & & 2 months & 4 months & 6 months & \\
\hline Systolic BP $(\mathrm{mmHg})$ & $127.92 \pm 18.15$ & $125.83 \pm 16.63$ & $126.67 \pm 14.67$ & $127.08 \pm 16.44$ & 0.03 & 0.99 \\
Diastolic BP (mmHg) & $77.92 \pm 9.64$ & $77.50 \pm 9.17$ & $76.67 \pm 8.07$ & $77.08 \pm 7.53$ & 0.05 & 0.99 \\
Pulse (pulses/minute) & $73.08 \pm 12.13$ & $73.08 \pm 9.65$ & $74.00 \pm 8.69$ & $73.33 \pm 8.86$ & 0.02 & 1.00 \\
Visual acuity & $0.79 \pm 0.27$ & $0.86 \pm 0.28$ & $0.86 \pm 0.28$ & $0.83 \pm 0.23$ & $9.88^{\mathrm{b}}$ & 0.95 \\
IOP (mmHg) & $14.33 \pm 2.39$ & $14.29 \pm 2.53$ & $14.70 \pm 2.07$ & $14.87 \pm 1.84$ & 0.32 & 0.81 \\
VCDR & $0.87 \pm 0.17$ & $0.87 \pm 0.17$ & $0.87 \pm 0.17$ & $0.87 \pm 0.17$ & 0.00 & 1.00 \\
\hline
\end{tabular}

a Data are expressed as mean \pm SD.

b Kruskal-Wallis test.

factors such as elevated IOP, [17-19] decreased neurotrophin support, ${ }^{[20,21]}$ glutamate-associated excitotoxicity, ${ }^{[22-24]}$ hypoperfusion, and vasospasm $^{[25-27]}$ have been implicated in ganglion cell death in glaucoma. Neuroprotective strategies have focused on mitigating these risk factors associated with RGC loss in glaucoma.

EBHM is classified as a Chinese herbal medicine. EBHM is absorbed in the intestine through passive diffusion, and a linear relationship exists between absorption and blood concentration of EBHM over the concentration range of 50-400 $\mu \mathrm{g} / \mathrm{mL}$. ${ }^{[28]}$ After absorption, EBHM is mainly distributed in kidneys, heart, liver, and brain, and the mean retention time is $180.70 \pm 54.38$ minutes. ${ }^{[28]}$ The main active constituents of EBHM extract include 4 '-hydroscutellarein, 4'-hydrobaicalein-7- $\beta$ D-plamyagin, 4'-hydroscutellarein-7- $\beta$-D-glycuron methyl ester, pyromeconic acid, and several kinds of flavones and flavonoids. ${ }^{[29-32]}$ Pharmacologic studies have demonstrated that EBHM can dilate the blood vessels, reduce blood vessel resistance, increase blood flow, improve microcirculation, and inhibit thrombosis. ${ }^{[9,10]}$ Although it is not known which components of EBHM are attributed to the specific effects, it has been suggested that the combined activity and a certain interdependency of several active constituents of EBHM extract are responsible for its beneficial effects. ${ }^{[33]}$ It is most likely that the protective and

Table IV. Comparison of visual field indices between pre- and post-treatment periods in the placebo and Erigeron breviscapus (vant.) Hand. Mazz. (EBHM) groups ${ }^{a}$

\begin{tabular}{|c|c|c|c|c|c|c|c|c|}
\hline & \multicolumn{4}{|c|}{ Placebo group $(n=20)$} & \multicolumn{4}{|c|}{ EBHM group $(n=20)$} \\
\hline & pre-treatment & post-treatment & $\mathrm{t}$-test value & $p$-value & pre-treatment & post-treatment & t-test value & $p$-value \\
\hline \multicolumn{9}{|l|}{2 months } \\
\hline $\mathrm{MD}(\mathrm{dB})$ & $11.97 \pm 7.32$ & $12.55 \pm 7.25$ & -1.26 & 0.22 & $11.44 \pm 8.84$ & $10.98 \pm 8.67$ & 1.43 & 0.17 \\
\hline$M S(d B)$ & $14.91 \pm 7.60$ & $14.32 \pm 7.55$ & 1.26 & 0.22 & $15.48 \pm 8.46$ & $15.91 \pm 8.27$ & -1.35 & 0.19 \\
\hline $\operatorname{LV}\left(\mathrm{dB}^{2}\right)$ & $47.70 \pm 25.06$ & $54.00 \pm 27.58$ & -1.23 & 0.23 & $42.88 \pm 35.82$ & $45.70 \pm 38.06$ & -1.18 & 0.25 \\
\hline \multicolumn{9}{|l|}{4 months } \\
\hline $\mathrm{MD}(\mathrm{dB})$ & $11.97 \pm 7.32$ & $11.45 \pm 7.19$ & 0.97 & 0.35 & $11.44 \pm 8.84$ & $10.76 \pm 8.51$ & 1.73 & 0.10 \\
\hline$M S(d B)$ & $14.91 \pm 7.60$ & $15.41 \pm 7.42$ & -0.92 & 0.37 & $15.48 \pm 8.46$ & $16.13 \pm 8.11$ & -1.64 & 0.12 \\
\hline $\mathrm{LV}\left(\mathrm{dB}^{2}\right)$ & $47.70 \pm 25.06$ & $49.49 \pm 25.14$ & -0.59 & 0.57 & $42.88 \pm 35.82$ & $42.13 \pm 34.78$ & 0.53 & 0.60 \\
\hline \multicolumn{9}{|l|}{6 months } \\
\hline $\mathrm{MD}(\mathrm{dB})$ & $11.97 \pm 7.32$ & $11.65 \pm 7.13$ & 0.75 & 0.46 & $11.44 \pm 8.84$ & $9.87 \pm 8.53$ & 3.80 & $<0.01$ \\
\hline $\mathrm{MS}(\mathrm{dB})$ & $14.91 \pm 7.60$ & $15.17 \pm 7.45$ & -0.59 & 0.56 & $15.48 \pm 8.46$ & $16.99 \pm 8.13$ & -3.71 & $<0.01$ \\
\hline $\mathrm{LV}\left(\mathrm{dB}^{2}\right)$ & $47.70 \pm 25.06$ & $50.32 \pm 25.80$ & -0.91 & 0.37 & $42.88 \pm 35.82$ & $42.54 \pm 35.93$ & 0.11 & 0.92 \\
\hline
\end{tabular}


Table V. Comparison of visual field indices of moderate and late glaucoma patients between pre- and post-treatment periods in the placebo and Erigeron breviscapus (vant.) Hand. Mazz. (EBHM) groups ${ }^{a}$

\begin{tabular}{|c|c|c|c|c|c|c|c|c|}
\hline & \multicolumn{4}{|c|}{ Placebo group $(n=15)$} & \multicolumn{4}{|c|}{ EBHM group $(n=12)$} \\
\hline & pre-treatment & post-treatment & t-test value & $p$-value & pre-treatment & post-treatment & t-test value & $\mathrm{p}$-value \\
\hline \multicolumn{9}{|l|}{2 months } \\
\hline $\mathrm{MD}(\mathrm{dB})$ & $14.23 \pm 6.92$ & $15.02 \pm 6.42$ & -1.45 & 0.17 & $16.76 \pm 7.42$ & $15.78 \pm 7.90$ & 3.28 & 0.01 \\
\hline $\mathrm{MS}(\mathrm{dB})$ & $12.63 \pm 7.24$ & $11.81 \pm 6.79$ & 1.50 & 0.16 & $10.24 \pm 6.69$ & $11.19 \pm 7.17$ & -3.13 & 0.01 \\
\hline $\mathrm{LV}\left(\mathrm{dB}^{2}\right)$ & $52.14 \pm 24.74$ & $60.93 \pm 26.86$ & -1.32 & 0.21 & $59.51 \pm 35.27$ & $63.20 \pm 36.58$ & -1.32 & 0.21 \\
\hline \multicolumn{9}{|c|}{4 months } \\
\hline $\mathrm{MD}(\mathrm{dB})$ & $14.23 \pm 6.92$ & $13.68 \pm 6.70$ & 0.82 & 0.43 & $16.76 \pm 7.42$ & $15.37 \pm 7.94$ & 2.87 & 0.02 \\
\hline $\mathrm{MS}(\mathrm{dB})$ & $12.63 \pm 7.24$ & $13.15 \pm 6.96$ & -0.77 & 0.46 & $10.24 \pm 6.69$ & $11.60 \pm 7.27$ & -2.80 & 0.02 \\
\hline $\mathrm{LV}\left(\mathrm{dB}^{2}\right)$ & $52.14 \pm 24.74$ & $56.15 \pm 21.95$ & -1.12 & 0.28 & $59.51 \pm 35.27$ & $56.73 \pm 35.10$ & 0.52 & 0.62 \\
\hline \multicolumn{9}{|c|}{6 months } \\
\hline $\mathrm{MD}(\mathrm{dB})$ & $14.23 \pm 6.92$ & $14.03 \pm 6.43$ & 0.47 & 0.65 & $16.76 \pm 7.42$ & $14.42 \pm 8.08$ & 4.56 & $<0.01$ \\
\hline $\mathrm{MS}(\mathrm{dB})$ & $12.63 \pm 7.24$ & $12.75 \pm 6.83$ & -0.28 & 0.79 & $10.24 \pm 6.69$ & $12.53 \pm 7.36$ & -4.49 & $<0.01$ \\
\hline $\operatorname{LV}\left(\mathrm{dB}^{2}\right)$ & $52.14 \pm 24.74$ & $57.74 \pm 21.99$ & -1.84 & 0.09 & $59.51 \pm 35.27$ & $59.83 \pm 34.65$ & -0.07 & 0.95 \\
\hline
\end{tabular}

a Data are expressed as mean \pm SD.

$\mathbf{L V}=$ loss variance; $\mathbf{M D}=$ mean defect; $\mathbf{M S}=$ mean sensitivity.

rescuing effects of EBHM extract are attributable to its ability to improve microcirculation. Bastianetto et al. ${ }^{[34]}$ reported that the flavonoid fraction strongly inhibited both the toxicity and the free radical accumulation induced by sodium nitroprusside and/or 3-morpholinosydnonimine. EBHM extract contains several kinds of flavones and flavonoids; therefore, it may also have the effect of inhibiting the toxicity and the free radical accumulation induced by glaucomatous neuropathy. The present study shows that the visual field indices were significantly improved (MD decrease, MS increase) after 6 months of EBHM treatment, and the improvement of visual field indices with EBHM treatment was more significant in the moderate and late glaucoma patients.

It is well known that elevated IOP is the most important factor for glaucomatous optic neuropathy; thus, lowering IOP is the most effective and commonly used therapeutic approach in glau- coma management. The present study demonstrates that the mean IOP in the two groups was less than $15 \mathrm{mmHg}$ (EBHM group $14.33 \mathrm{mmHg}$, placebo group $13.71 \mathrm{mmHg}$ ), and there was no significant difference in IOP between the two groups during the treatment period. Therefore, it has been shown that EBHM is effective in rescuing glaucomatous optic neuropathy while maintaining IOP at normal or below normal levels.

The present study also shows that the visual field improvement rate in the EBHM group was higher than that in the placebo group after 6 months of treatment; however, it did not reach statistical significance, which may be attributed to the small number of patients. No treatmentrelated adverse effects were observed.

In summary, the results of this study suggested that EBHM extract may have a partially protective effect on the visual field of glaucoma patients with controlled IOP. Further studies are

Table VI. Comparison of visual field improvement rates between the placebo and Erigeron breviscapus (vant.) Hand. Mazz. (EBHM) groups

\begin{tabular}{|c|c|c|c|c|c|c|c|c|c|c|}
\hline & \multicolumn{4}{|c|}{ Placebo group $(n=20)$} & \multicolumn{4}{|c|}{ EBHM group $(n=20)$} & \multirow[t]{2}{*}{$\chi^{2}$} & \multirow[t]{2}{*}{$\mathrm{p}$-Value } \\
\hline & $\operatorname{IMP}(\mathrm{n})$ & $\mathrm{NC}(\mathrm{n})$ & RET (n) & IMP rate $(\%)$ & IMP (n) & $\mathrm{NC}(\mathrm{n})$ & RET (n) & IMP rate $(\%)$ & & \\
\hline 2 months & 2 & 15 & 3 & 10 & 2 & 18 & 0 & 10 & 1.13 & 0.57 \\
\hline 4 months & 5 & 13 & 2 & 25 & 3 & 17 & 0 & 15 & 3.03 & 0.22 \\
\hline 6 months & 3 & 16 & 1 & 15 & 7 & 13 & 0 & 35 & 2.91 & 0.23 \\
\hline
\end{tabular}


Table VII. Comparison of visual field improvement rates of moderate and late glaucoma patients between the placebo and Erigeron breviscapus (vant.) Hand. Mazz. (EBHM) groups

\begin{tabular}{|c|c|c|c|c|c|c|c|c|c|c|}
\hline & \multicolumn{4}{|c|}{ Placebo group $(n=15)$} & \multicolumn{4}{|c|}{ EBHM group $(n=12)$} & \multirow[t]{2}{*}{$\chi^{2}$} & \multirow[t]{2}{*}{ p-Value } \\
\hline & $\operatorname{IMP}(n)$ & $N C(n)$ & RET (n) & IMP rate $(\%)$ & $\operatorname{IMP}(n)$ & NC (n) & RET (n) & IMP rate $(\%)$ & & \\
\hline 2 months & 1 & 11 & 3 & 6.67 & 1 & 11 & 0 & 8.33 & 2.70 & 0.26 \\
\hline 4 months & 4 & 9 & 2 & 26.67 & 3 & 9 & 0 & 25.0 & 1.83 & 0.40 \\
\hline 6 months & 2 & 12 & 1 & 13.33 & 6 & 6 & 0 & 50.0 & 4.73 & 0.09 \\
\hline
\end{tabular}

needed to determine the safety and effectiveness of long-term EBHM treatment.

\section{Acknowledgments}

We thank Xiangya Pharmaceutical Co. for providing the EBHM and placebo tablets for the study. None of the authors had proprietary interest in the development and marketing of any products mentioned in the article. This research was supported by Shanghai Leading Academic Discipline Project (No. S30205).

\section{References}

1. Anderson DR. Glaucoma: the damage caused by pressure. XLVI Edward Jackson memorial lecture. Am J Ophthalmol 1989; 108: 485-95

2. Bathija R, Gupta N, Zangwill L, et al. Changing definition of glaucoma. J Glaucoma 1998; 7: 165-9

3. Foster PJ, Buhrmann R, Quigley HA, et al. The definition and classification of glaucoma in prevalence surveys. Br J Ophthalmol 2002; 86: 238-42

4. Palmberg P. Risk factors for glaucoma progression: where does intraocular pressure fit in? Arch Ophthalmol 2001; 119: $897-8$

5. Leske MC, Heijl A, Hussein M, et al. Factors for glaucoma progression and the effect of treatment: the early manifest glaucoma trail. Arch Ophthalmol 2003; 121: 48-56

6. Collaborative Normal-Tension Glaucoma Study Group. The effectiveness of intraocular pressure reduction in the treatment of normal-tension glaucoma. Am J Ophthalmol 1998; 126: 498-505

7. The AIGS investigators. Advanced glaucoma intervention study: 7. The relationship between control of intraocular pressure and visual field deterioration. Am J Ophthalmol 2000; 130: 429-40

8. Gao M, Gu M, Liu CZ. Two-step purification of scutellarin from Erigeron breviscapus (vant.) hand-mazz by high-speed counter-current chromatography. J Chromatogr B Analyt Technol Biomed Life Sci 2006; 838: 139-43

9. Zhou QS, Zhao YM, Bai X, et al. Effect of new-breviscapine on fibrinolysis and anticoagulation of human vascular endothelial cells. Zhongguo Yao Li Xue Bao 1992; 13: 239-42

10. Wang ZY, Chen DC, He Y, et al. Differential effects of new breviscapine on arachidonic acid metabolisms in blood cells and endothelial cells. Zhongguo Yao Li Xue Bao 1993; 14: 148-51
11. Jia L, Liu Z, Luo X, et al. The effect of Qing Guang Kang on the metabolism of retinal ganglion cells of rats after artificial acute high intraocular pressure. Zhonghua Yan Ke Za Zhi 1995; 31: 129-31

12. Zhu Y, Jiang Y, Liu Z, et al. The affect of Erigeron breviscapus (vant.) hand-mazz on axoplasmic transport of optic nerve in rats with experimentally elevated intraocular pressure. Zhonghua Yan Ke Za Zhi 2000; 36: 289-91

13. European Glaucoma Society. Terminology and guidelines for glaucoma. 2nd ed. Savona: Dogma, 2003: 6-8

14. Iester M, Swindale NV, Mikelberg FS. Sector-based analysis of optic nerve head shape parameters and visual field indices in healthy and glaucomatous eyes. J Glaucoma 1997; 6: 371-6

15. Caprioli J. The contour of the juxtapapillary nerve fiber layer in glaucoma. Ophthalmology 1990; 97: 358-66

16. Hodapp E, Parrish II R, Anderson D. Clinical decisions in glaucoma. St. Louis (MO): Mosby-Year Book Inc., 1993: 11-63

17. Heijl A, Leske MC, Bengtsson B, et al. Reduction of intraocular pressure and glaucoma progression: results from the early manifest glaucoma trial. Arch Ophthalmol 2002; 120: $1268-79$

18. Collaborative Normal-Tension Glaucoma Study Group. The effectiveness of intraocular pressure reduction in the treatment of normal-tension glaucoma: Collaborative Normal-Tension Glaucoma Study Group. Am J Ophthalmol 1998; 126: 498-505

19. Collaborative Normal-Tension Glaucoma Study Group. Comparison of glaucomatous progression between untreated patients with normal tension glaucoma and patients with therapeutically reduced intraocular pressure: Collaborative Normal-Tension Glaucoma Study Group. Am J Ophthalmol 1998; 126: 487-97

20. Quigley HA, McKinnon SJ, Zack DJ, et al. Retrograde axonal transport of BDNF in retinal ganglion cells is blocked by acute IOP elevation in rats. Invest Ophthamol Vis Sci 2000; 41: 3460-6

21. Pease ME, McKinnon SJ, Quigley HA, et al. Obstructed axonal transport of BDNF and its receptor TRKB in experimental glaucoma. Invest Ophthamol Vis Sci 2000; 41: 764-74

22. Vorwerk CK, Gorla MS, Dreyer EB. An experimental basis for implicating excitotoxicity in glaucomatous optic neuropathy. Surv Ophthalmol 1999; 43 Suppl. 1: S142-50

23. Dreyer EB, Zurakowski D, Schumer RA, et al. Elevated glutamate levels in the vitrous body of humans and monkeys with glaucoma. Arch Ophthalmol 1996; 114: 299-305

24. Vorwerk CK, Lipton SA, Zurakowski D, et al. Chronic lowdose glutamate is toxic to retinal ganglion cells: toxicity 
blocked by memantine. Invest Ophthamol Vis Sci 1996; 37: 1618-24

25. Flammer J, Orgul S, Costa VP, et al. The impact of ocular blood flow in glaucoma. Prog Retin Eye Res 2002; 21: 359-93

26. Hayreh SS. The blood supply of the optic nerve head and the evaluation of it-myth and reality. Prog Retin Eye Res 2001; 20: $563-93$

27. Tielsch JM, Katz J, Sommer A, et al. Hypertension, perfusion pressure, and primary open-angle glaucoma: a populationbased assessment. Arch Ophthalmol 1995; 113: 216-21

28. Dongmei Y, Liyuan K, Limin H. Pharmacokinetic studies of Erigeron breviscapus. World Science and TechnologyModernization of Traditional Chinese Medicine and Materia Medica 2009; 11 (2): 299-303

29. Zhang RW, Yang SY, Lin YY. Studies on chemical constituents of dengzhanhua (Erigeron breviscapus (vant.) hand-mazz): I. The isolation and identification of pyromeconic acid and a new glucoside (author's translation). Yao Xue Xue Bao 1981; 16: 68-9

30. Zhang WD, Chen WS, Wang YH, et al. Studies on flavone constituents of Erigeron breviscapus (vant.) hand.-mazz. Zhongguo Zhong Yao Za Zhi 2000; 25: 536-8
31. Zhang WD, Ha TB, Chen WS, et al. Study on the structure and activity of new phenolic acid compounds from Erigeron breviscapus. Yao Xue Xue Bao 2001; 36: 360-3

32. Zhang WD, Chen WS, Wang YH, et al. Two new glycosides from Erigeron breviscapus (vant.) hand.-mazz. Zhongguo Zhong Yao Za Zhi 2001; 26: 689-90

33. Chu Q, Wu T, Fu L, et al. Simultaneous determination of active ingredients in Erigeron breviscapus (vant.) handmazz. by capillary electrophoresis with electrochemical detection. J Pharm Biomed Anal 2005; 37: 535-41

34. Bastianetto S, Zheng WH, Quirion R. The Ginkgo biloba extract (EGb 761) protects and rescues hippocampal cells against nitric oxide-induced toxicity: involvement of its flavonoid constituents and protein kinase C. J Neurochem 2000; 74: 2268-77

Correspondence: Dr Yisheng Zhong, Department of Ophthalmology, Ruijin Hospital Affiliated Medical School, Shanghai Jiaotong University, 197 Ruijin No. 2 Road, 200025 Shanghai, China.

E-mail: yszhong68@yahoo.com.cn 